\title{
Corticosteroids prevent the progression of autoimmune pancreatitis to chronic pancreatitis
}

Yasuhiro Kuraishi $^{1}$, Takeshi Uehara ${ }^{2}$, Takayuki Watanabe ${ }^{1}$, Norihiro Ashihara ${ }^{1}$, Makiko Ozawa ${ }^{1}$, Keita Kanai ${ }^{1}$, Shigeyuki

$\mathrm{Kawa}^{3}$

${ }^{1}$ Department of Gastroenterology, Shinshu University School of Medicine, Matsumoto, Nagano, Japan

${ }^{2}$ Department of Laboratory Medicine, Shinshu University School of Medicine, Matsumoto, Nagano, Japan

${ }^{3}$ Department of Internal Medicine, Matsumoto Dental University, Shiojiri, Nagano, Japan

\section{Short title: Long-term effect of steroids in AIP}

\section{Corresponding author:}

Takeshi Uehara, M.D., Department of Laboratory Medicine, Shinshu University School of Medicine, 3-1-1 Asahi,

Matsumoto 390-8621, Japan

E-mail: tuehara@shinshu-u.ac.jp; Tel: +81-263-37-2805; Fax: +81-263-34-5316 


\section{Abstract}

Background/Objectives: Patients with autoimmune pancreatitis (AIP) sometimes progress to chronic pancreatitis (CP). We evaluated the ability of corticosteroids to prevent the progression to $\mathrm{CP}$.

Methods: We defined patients with definitive findings of CP (stones in the main pancreatic duct [MPD] or multiple pancreatic calcifications) as having severe calcification (SC). A total of 145 AIP patients were enrolled. We measured the duration between AIP diagnosis and SC development and retrospectively compared the time to SC development between patients with and without steroids. Multivariate analysis for factors associated with SC were performed.

Results: Nineteen (13\%) patients progressed to SC. Since 95 patients had pancreatic head swelling and SC was found in these patients only, our analysis focused mainly on these at-risk populations. In Kaplan-Meier analysis limited to patients with pancreatic head swelling, the incidence of SC was significantly lower in patients with steroids than in those without (hazard ratio $[\mathrm{HR}] 0.18,95 \%$ confidence interval [CI] 0.07-0.52; $<<0.001$ ). Multivariate testing of patients with pancreatic head swelling confirmed that steroid therapy was significantly associated with a lower incidence of SC (HR $0.11,95 \%$ CI 0.03-0.34; $<<0.001)$, while MPD dilation at AIP diagnosis was related to a higher incidence of SC (HR 4.02, 95\% CI 1.43$11.7 ; \mathrm{p}=0.009)$.

Conclusions: Corticosteroids appeared to prevent progression to CP in AIP patients, especially in those with pancreatic head swelling. Patients with both pancreatic head swelling and MPD dilation at diagnosis have a higher incidence of progression to CP. Steroid therapy is suggested for these high-risk cases.

Key words: autoimmune pancreatitis; chronic pancreatitis; pancreatic calcification; steroid therapy 


\section{Introduction}

Type 1 autoimmune pancreatitis (AIP in the present paper) is a distinct type of pancreatitis displaying elevated serum IgG4

levels and characteristic image findings of pancreatic enlargement and irregular narrowing of the main pancreatic duct (MPD) that responds well to steroid therapy ${ }^{1-3}$. The pancreatic duct narrowing in AIP stems from dense lymphoplasmacytic infiltration with extensive fibrosis mainly composed of IgG4-positive plasma cells around the pancreatic duct ${ }^{4}$, which are improved both morphologically and histologically by steroid treatment ${ }^{5}$.

Patients with ordinary chronic pancreatitis (CP), such as alcoholic pancreatitis, frequently exhibit the most common and characteristic complication of pancreatic stones. Pancreatic stone formation is caused by the hypersecretion of protein from acinar cells and pancreatic juice stasis ${ }^{6}$. In CP, pancreatic stones and MPD stricture induce pancreatic juice stasis in the upstream pancreatic duct and increase pancreatic intraductal pressure, which worsens the morphological and functional disease state ${ }^{7}$. On the other hand, AIP was earlier believed as free from pancreatic stones and transformation into confirmed CP resembling alcoholic pancreatitis because it was considered a reversible and acute disorder due to histologically dense lymphoplasmacytic infiltration and a favorable response to steroid therapy ${ }^{3}$. However, it has become apparent that some AIP patients experience pancreatic stone formation and pancreatic atrophy over a long-term course ${ }^{8-11}$, suggesting that AIP can also progress to a state similar to ordinary CP. A prior study on serum IgG4 levels in $175 \mathrm{CP}$ patients who had been diagnosed before 1995, when the concept of AIP was first proposed, showed elevated IgG4 in $7.4 \%$ patients ${ }^{12}$, which indicated that some cases may have been attributable to AIP.

The frequency of pancreatic calcification in AIP reportedly ranges from $5 \%$ to $40 \%{ }^{11,13-16}$. We previously described that 
pancreatic stone formation was significantly associated with pancreatic head swelling and narrowing of both Wirsung's and Santorini's ducts in the pancreatic head region at diagnosis ${ }^{15}$. Moreover, we investigated the risk factors of progression to $\mathrm{CP}$ fulfilling the Japanese clinical diagnostic criteria, including stones in the pancreatic duct and multiple calcifications distributed throughout the entire pancreas, in AIP patients who underwent long-term follow-up ${ }^{10}$. Multivariate analysis revealed that pancreatic head swelling and MPD non-narrowing in the pancreatic body were significant independent risk factors for the development of confirmed CP. This close association of pancreatic head swelling with pancreatic stone formation was confirmed in a Japanese multicenter study ${ }^{14}$. In experimental canine models of MPD stenosis from incomplete MPD ligation, partial outflow obstruction of the pancreatic duct induced the formation of pancreatic stones ${ }^{17}$, ${ }^{18}$. Collectively, the above findings indicate the underlying mechanism of AIP progression to $\mathrm{CP}$ to be pancreatic juice stasis induced by pancreatic head swelling over a long-term period ${ }^{10,11,15}$. MPD narrowing accompanied by pancreatic head swelling causes the upstream stasis of pancreatic juice, which leads to the formation of pancreatic stones and ultimately CP.

Alcoholic and other forms of ordinary $\mathrm{CP}$ are irreversible conditions that result in pancreatic exocrine and endocrine insufficiency and often display severe complications, such as pancreatic cancer and pseudocysts ${ }^{19}$. AIP patients with pancreatic calcification and atrophy have also been reported to suffer from pancreatic exocrine and endocrine abnormalities ${ }^{8,9,20}$. Moreover, some AIP patients exhibit pseudocysts ${ }^{21}$ or pancreatic cancer ${ }^{22,23}$ similar to those in ordinary CP.

AIP patients respond well to corticosteroid therapy in the short term, with pancreatic exocrine and endocrine function being improved somewhat by the treatment ${ }^{16,24-26}$. However, the long-term influence of steroids on AIP progression to CP 
remains unclear. Considering the favorable response to steroid therapy for improving MPD narrowing, pancreatic swelling, and presumably pancreatic juice stasis in AIP patients, steroids are also suspected to prevent the progression to CP. We herein aimed to clarify the efficacy of corticosteroid treatment for preventing AIP progression to $\mathrm{CP}$ and the appropriate indications for steroid therapy towards effective long-term AIP management.

\section{Methods}

This study adhered to the current ethical guidelines of the Declaration of Helsinki and was conducted in accordance with the requirements of the Institutional Review Board of Shinshu University School of Medicine (approval number: 4019).

\section{Patients}

The medical records of AIP patients who were treated between January 1996 and November 2018 at Shinshu University Hospital in Matsumoto, Japan, were reviewed retrospectively. Of them, a total of 145 AIP patients (107 men and 38 women, median age: 67 years [range: $38-92$ years]) who had been followed for at least 1 year were enrolled. Median follow-up duration was 78 months (range: 14-284 months). All patients had type 1 AIP. The diagnosis of AIP was conducted according to the International Consensus Diagnostic Criteria ${ }^{27}$.

Among the 145 AIP patients, 102 (70\%) had received steroid therapy based on the Japanese consensus guidelines for $\mathrm{AIP}^{28}$. The initial oral prednisolone dose for remission induction was $0.6 \mathrm{mg} / \mathrm{kg} / \mathrm{day}$ for approximately 4 weeks, which was then tapered by $5 \mathrm{mg}$ every $1-2$ weeks until a daily dose of $5 \mathrm{mg} /$ day. In 74 of 85 patients $(87 \%)$ who had been followed for at least 3 years, maintenance steroid therapy of $5 \mathrm{mg} /$ day was conducted for more than 3 years. 


\section{Evaluation for transformation into $\mathrm{CP}$}

We examined AIP patients for progression to $\mathrm{CP}$ based on the revised Japanese clinical diagnostic criteria for $\mathrm{CP}^{29}$. Cases with image findings of stones in the MPD or multiple calcifications distributed throughout the pancreas, which were definite findings of CP in the clinical diagnostic criteria, were defined as severe calcification (SC) in this study. Accordingly, the development of SC indicated progression to $\mathrm{CP}$ in terms of image findings. We evaluated the presence of SC retrospectively using cross-sectional computed tomography (CT) images for each patient. CT-based follow-up was performed at least once a year for almost all subjects.

The following clinicodemographic factors potentially associated with pancreatic calcification were assessed: sex, age, drinking and smoking habits, abdominal/back pain, obstructive jaundice, pancreatic swelling, other organ involvements found in IgG4-related disease, elevations in serological IgG, IgG4, or amylase, relapse, and MPD dilation. All factors apart from relapse were analyzed at the time of AIP diagnosis. Other organ involvements were limited to multiple biliary strictures, salivary/lachrymal gland enlargement, retroperitoneal fibrosis, tubulointerstitial nephritis, and interstitial pneumonitis. Similarly to past reports ${ }^{10,15}$, pancreatic swelling was evaluated based on the Haaga criteria ${ }^{30}$ or a marked reduction in size after steroid therapy. Serological values of $\mathrm{IgG} \geq 1800 \mathrm{mg} / \mathrm{dL}, \mathrm{IgG} 4 \geq 135 \mathrm{mg} / \mathrm{dL}$, and amylase $\geq 130 \mathrm{IU} / \mathrm{L}$ were regarded as positive for serum elevation. Relapse was defined as pancreatic lesion recurrence after remission during the study course. The presence of MPD dilation $(\geq 3 \mathrm{~mm})$ at 1 or more pancreatic areas, mainly in the pancreatic body and tail, was evaluated using endoscopic retrograde cholangiopancreatography and magnetic resonance cholangiopancreatography. 
We measured the duration between AIP diagnosis and SC development and compared the time to SC development between patients with and without steroid therapy to evaluate the effectiveness of corticosteroid treatment for preventing the progression to $\mathrm{CP}$.

\section{Statistical analysis}

For clinicodemographic characteristics, the variables for each patient were expressed as the number and percentage. Pearson's chi-squared tests were adopted to test for differences between subgroups of patients. The time to SC development in AIP patients was calculated using the Kaplan-Meier method, and the difference in those rates was compared by the logrank test. Univariate and multivariate analyses for factors associated with SC were performed using a Cox proportional hazard regression model. Variables that reached a p-value of 0.2 in univariate analysis were further considered in multivariate analysis. A p-value of $<0.05$ was regarded as statistically significant. All statistical analyses were performed using JMP Statistics software version 13 (JMP, Tokyo, Japan).

\section{Results}

\section{Characteristics of AIP patients with severe pancreatic calcification}

Of the 145 patients, diffuse pancreatic swelling and pancreatic head swelling were observed in 85 patients (59\%) and 96 patients (66\%), respectively. Diffuse pancreatic swelling was defined as swelling in more than two thirds of the pancreas. Patients with pancreatic head swelling included not only cases of pancreatic head swelling, but also those of body or tail swelling in addition to head swelling. Respective median serum IgG and IgG4 levels were $1976 \mathrm{mg} / \mathrm{dL}$ (range: 379-7142 
$\mathrm{mg} / \mathrm{dL}$ ) and $459 \mathrm{mg} / \mathrm{dL}$ (range: $4-3660 \mathrm{mg} / \mathrm{dL}$ ). Thirty-four patients (23\%) experienced at least 1 pancreatic lesion relapse.

MPD dilation at diagnosis was observed in 44 patients (30\%).

Nineteen of 145 recruited patients (13\%) exhibited SC displaying the characteristic image findings that fulfilled the clinical diagnostic criteria for CP. SC was detected at the initial diagnosis of AIP in 2 patients and during subsequent followup in 17 patients (Figure 1a). Specifically, pancreatic duct stones were seen in 12 patients and multiple calcifications distributed throughout the pancreas were found in 15 patients, with overlap in some patients (Figure 1b). Pancreatic head swelling and MPD dilation at AIP diagnosis were observed in 18 patients (95\%) and 10 patients (53\%), respectively.

To assess pancreatic exocrine function in AIP with SC, we measured fecal elastase-1 concentration (FEC) ${ }^{31}$ in 11 of 19 patients experiencing SC during follow-up. Exocrine insufficiency was defined as FEC $<200 \mu \mathrm{g} / \mathrm{g}$. Median FEC level was $68 \mu \mathrm{g} / \mathrm{g}$ (range: $5-520 \mu \mathrm{g} / \mathrm{g}$ ) and the rate of patients with exocrine insufficiency was $73 \%(8 / 11)$. Regarding endocrine function in patients with SC, 17 patients (90\%) suffered from diabetes mellitus, 7 of which required insulin administration. The rate of patients with insulin secretory insufficiency as defined by fasting immunoreactive insulin $<5.0 \mu \mathrm{U} / \mathrm{mL}$ or fasting C-peptide reactivity $<0.6 \mathrm{ng} / \mathrm{dL}$ was $41 \%$ (7/17). With regard to complications accompanying SC development, pseudocysts were found in 5 patients (26\%) and pancreatic attacks caused by incarceration of pancreatic stones were seen in 2 patients (10\%), with no patients exhibiting pancreatic cancer. Extracorporeal shock wave lithotripsy (ESWL) and surgical resection were respectively performed on 8 and 1 patients as interventional therapy for pancreatic stones.

\section{Evaluation of steroid therapy effects}

To assess the efficacy of steroid therapy to prevent AIP progression to $\mathrm{CP}$, the incidence of SC was compared between 
cases with and without steroids using Kaplan-Meier testing. The 2 patients already complicated with SC at the diagnosis of AIP were excluded. One was of a heavy-drinking patient without pancreatic head swelling, and the other had pancreatic head swelling. The remaining 143 patients were enrolled for the evaluation of steroid effectiveness. The baseline characteristics of patients with and without steroid therapy are compared in Table 1 . The prevalences of abdominal/back pain, obstructive jaundice, pancreatic head swelling, and serum IgG elevation in patients receiving steroids were significantly higher than in those without steroids, which indicated that corticosteroid therapy was performed more frequently for cases of problematic symptoms or an active disease state. Three patients not given steroids at AIP diagnosis later received steroids upon SC detection. These patients were classified into the non-steroid treated group. In 1 case, pancreatic calcification was seen to improve with steroids, whereas pancreatic stones continued to increase slowly in number and size in the remaining 2 cases. The clinical and radiological course of the improved case is presented in Figure 2. In the Kaplan-Meier evaluation of all 143 AIP patients, the incidence of SC was significantly lower in cases with steroids than in those without (hazard ratio: $0.33,95 \%$ confidence interval [CI] 0.12-0.94; $\mathrm{p}=0.025$, log-rank test) (Figure 3a).

\section{Evaluation of steroid therapy effects in cases with pancreatic head swelling}

Pancreatic head enlargement is a reported risk factor for AIP progression to CP that is associated with pancreatic juice stasis $^{10,11,14,15}$. Therefore, the 143 AIP patients were classified based on the presence or absence of pancreatic head swelling (Figure 4). Of the 95 patients with pancreatic head swelling, 77 were treated with steroid therapy and 18 were not, with 10 and 7 cases displaying SC, respectively. On the other hand, no patients experienced SC among the 48 cases without pancreatic head swelling at AIP diagnosis. Therefore, AIP patients without pancreatic head swelling were not considered 
to be at risk for SC development and excluded, leaving 95 patients with pancreatic head swelling for comparisons of the incidence of SC between cases with and without corticosteroids. Kaplan-Meier evaluation showed that the incidence of SC was significantly lower in cases with steroids (hazard ratio: $0.18,95 \%$ CI $0.07-0.52 ; \mathrm{p}<0.001$, log-rank test) (Figure $3 \mathrm{~b}$ ).

Multivariate analysis was performed on the incidence of SC in cases with pancreatic head swelling, which were considered a risk group for SC (Table 2). Univariate analysis revealed MPD dilation at diagnosis to be significantly associated with a higher incidence of SC and steroid therapy to be significantly related to a lower incidence. Patients aged $\geq 65$ years tended to have a higher incidence of SC. Multivariate analysis confirmed that steroid therapy was an independent factor contributing to a lower incidence of SC (hazard ratio: $0.11,95 \% \mathrm{CI} 0.03-0.34 ; \mathrm{p}<0.001$ ) and that MPD dilation at diagnosis was an independent factor associated with a higher SC incidence (hazard ratio: 4.02, 95\% CI 1.43-11.7; $\mathrm{p}=0.009$ ).

To identify the resistance factors to steroid therapy, the characteristics of 77 patients with pancreatic head swelling who received steroids were compared between cases with and without SC (Table 3). Relapse during follow-up was significantly associated with a higher incidence of SC $(\mathrm{p}=0.005)$.

\section{Discussion}

\section{Characteristics of patients with AIP progressing to CP}

The frequency of pancreatic calcification in AIP, which is the most common finding in CP, ranges from $5 \%$ to $40 \%{ }^{11,13-16}$, with discrepancies in reported frequencies caused by differences in follow-up period, disease activity, and therapeutic strategy. Here, although only cases of SC meeting the clinical diagnostic criteria for $\mathrm{CP}$ were enrolled, $13 \%$ of patients experienced SC during a long-term observation period, which suggested that the progression to $\mathrm{CP}$ was not rare in AIP. 
Most of the SC patients displayed exocrine and endocrine dysfunction similar to that in ordinary CP, and some exhibited problematic complications, such as pseudocysts and pancreatic attacks caused by pancreatic stone stasis. Thus, progressing to $\mathrm{CP}$ in AIP is a clinically serious problem. Marie et al. ${ }^{9}$ evaluated the clinical course of 44 AIP patients for a 3-year period following diagnosis and observed pancreatic morphological abnormalities such as calcification, atrophy, and duct irregularities in 35\% of cases, as well as exocrine and endocrine insufficiency in 34\% and 39\% of cases, respectively. Kanai et al. ${ }^{8}$ examined exocrine and endocrine function in 92 AIP patients by comparing cases with and without severe pancreatic calcification. Exocrine dysfunction was found in $39 \%$ of the non-calcified group and $56 \%$ of the calcified group, while endocrine dysfunction was seen in $26 \%$ of the non-calcified group and $31 \%$ of the calcified group. Indeed, AIP patients can sometimes progress to $\mathrm{CP}$ characterized by SC over a long-term course. In such progressed situations, exocrine and endocrine insufficiency and problematic complications such as pseudocysts and pancreatic attacks can occur as in ordinary CP. Therefore, appropriate management and treatment strategies are required for preventing the progression to CP in AIP.

\section{Evaluation of steroid therapy effects}

Kaplan-Meier testing revealed that patients receiving corticosteroid therapy had a significantly lower incidence of SC,

which indicated that steroids could prevent or delay the progression to CP in AIP. Since no patient without pancreatic head swelling displayed SC, we evaluated the incidence of SC only in patients with pancreatic head swelling as an at-risk population. Patients with steroid treatment had a remarkably lower incidence of SC and hazard ratio as compared with the former analysis, suggesting that steroid therapy targeting cases of pancreatic head swelling was more beneficial. Moreover, multivariate analysis confirmed steroid administration to reduce the risk of progression to CP in AIP patients with 
pancreatic head swelling.

MPD dilation at AIP diagnosis was also revealed as an independent factor associated with $\mathrm{SC}$ in multivariate analysis.

According to past reports ${ }^{10,11,14,15}$, pancreatic fluid stagnation from pancreatic head enlargement can induce AIP progression to CP. In agreement with this mechanism, no AIP patients without pancreatic head swelling experienced SC.

MPD dilation is presumed to reflect the pancreatic juice stasis induced duct narrowing accompanied by pancreatic head swelling. AIP shows a favorable response to steroid therapy, with dramatic and rapid improvement of lymphoplasmacytic cell infiltration and fibrosis to ameliorate pancreatic duct narrowing and pancreatic swelling 5 . Pancreatic juice stagnation is released by steroid treatment due to the improvement of MPD narrowing, which culminates in the prevention of progression to $\mathrm{CP}$.

We encountered a case of SC improvement after starting steroid therapy, which indicated that such treatment could not only prevent SC, but also ameliorate it. Tissue damage caused by AIP, including dense fibrosis, has been found to improve histologically by steroids ${ }^{32}$. Song et al. ${ }^{33}$ described that type IV collagen was preserved in AIP tissues in comparison with CP tissues. Since collagen type IV is an important component of the basement membrane that plays a role in scaffolding to ensure the accurate regeneration of tissue form during renewal and repair, the original structure of AIP tissue may be regenerated after tissue injury. Based on these findings, chronic stage AIP might be considered to have a different pathophysiology from that of ordinary $\mathrm{CP}$. Although ordinary $\mathrm{CP}$ is an irreversible condition, some $\mathrm{CP}$ cases originating from AIP may have the potential of tissue injury reversibility and regeneration; corticosteroid therapy may morphologically and histologically ameliorate the progressive condition of $\mathrm{CP}$ by relieving pancreatic juice stasis.

Among the patients receiving steroids, those suffering an AIP relapse had a significantly higher incidence of SC, which 
implicated relapse as a resistance factor to treatment. Relapse is a reported susceptibility factor for pancreatic stone formation ${ }^{13,34}$. A multinational survey showed that $46(7 \%)$ of 659 patients had pancreatic calcification and that this condition was closely associated with relapse ${ }^{13}$. Similarly to repeated pancreatic attacks in CP facilitating the progression of disease state, multiple recurrences of AIP presumably contribute to remnant pancreatic duct stricture, pancreatic juice stasis, and transformation to $\mathrm{CP}$.

\section{Indication for steroid therapy}

We previously examined the efficacy of endoscopic therapy and ESWL for pancreatic stones in 8 patients with chronic stage AIP and observed a lower rate of complete stone extraction from the MPD as compared with ordinary CP due to duct stenosis proximal to stones ${ }^{35}$. Since the therapeutic options for pancreatic stones are limited when AIP has progressed to $\mathrm{CP}$, better strategies for preventing the progression to $\mathrm{CP}$ are required. According to the Japanese Consensus Guidelines, the standard indication for steroid therapy is obstructive jaundice and any symptom, such as abdominal pain, as well as other organ involvement associated with IgG4-related disease. However, these criteria focus on only the short-term prognosis of AIP patients. Although there is insufficient consensus on the efficacy of steroid therapy on the long-term prognosis of AIP, our findings indicate an effectiveness of steroids for preventing the progression to CP. AIP patients with both pancreatic head swelling and MPD dilation at diagnosis had a particularly higher incidence of SC. Therefore, steroid therapy may be suggested for such high-risk cases to prevent disease progression.

As relapse was a significant risk factor for SC development in the steroid-treated cases, strategies to prevent relapse require further consideration. In a multicenter randomized control trial from Japan, relapse rate was significantly lower in 
patients with maintenance therapy who received prednisolone of 5-7.5 mg/day for 3 years than in those who discontinued the drug ${ }^{36}$. As we enrolled AIP patients who had been followed for more than 1 year and whose follow-up period was within 3 years, the effectiveness of maintenance therapy could not be fully examined. Maintenance therapy that can suppress relapse should be considered to prevent the progression to CP.

This investigation had several limitations. First, it was retrospective, contained a limited sample size, and was conducted at a single institution. The baseline characteristics of patients with and without steroid therapy varied with respect to a higher prevalence of abdominal/back pain, obstructive jaundice, pancreatic head swelling, and serum IgG elevation in the steroid-receiving group, which indicated that steroid treatment was more frequently performed in cases of problematic symptoms or an active disease state. Accordingly, multivariate analysis on the incidence of SC in cases with pancreatic head swelling was conducted to eliminate the influence of confounding as much as possible. Univariate analysis revealed that abdominal/back pain, obstructive jaundice, and serum IgG elevation were not remarkable factors contributing to AIP. Hence, symptoms and disease activity state, which were characteristics found more often in the steroid-receiving group, were not associated with progression to SC. We previously investigated the risk factors of pancreatic stone formation and progression to $\mathrm{CP}$ and revealed no significant associations between pancreatic stone formation and the degree of such published activity markers at AIP diagnosis as serum IgG, IgG, or complement or circulating immune complex ${ }^{10,15}$. However, since it is impossible to completely exclude the influence of bias caused by comparisons of different groups with and without steroid therapy, larger prospective trials are required to validate our results in the clinical setting. Second, we did not compare pancreatic exocrine or endocrine function between patients with and without steroids; the long-term effects of corticosteroids on pancreatic function remain unclear. Since steroid therapy could effectively halt progression to CP, this 
treatment was thought as effective for preventing pancreatic dysfunction in the long-term AIP course. According to earlier reports, the short-term effects of steroid therapy on exocrine function have consistently been favorable, whereas those on endocrine function are controversial, with studies showing recovery as well as worsening ${ }^{16,24-26,37}$. To fully elucidate the effectiveness of steroids on long-term AIP course, further investigations that include pancreatic function are necessary.

\section{Conclusions}

In conclusion, the present study revealed that corticosteroid therapy appeared effective for preventing the progression to $\mathrm{CP}$ in AIP patients, especially in those with pancreatic head swelling. Patients with CP originating from AIP exhibited exocrine and endocrine insufficiency and such complications as pseudocysts and pancreatic attacks. AIP patients with both pancreatic head swelling and MPD dilation at diagnosis have a higher incidence of progression to CP and may be indicated for steroid therapy. As relapse is a possible resistance factor to treatment, maintenance therapy is advised for avoiding potential relapse and conserving pancreatic function.

\section{Acknowledgment}

We thank Jane Charbonneau, DVM, from Edanz Group (www.edanzediting.com/ac) for editing a partial draft of this manuscript.

Conflict of interest: The authors declare that they have no conflicts of interest. 


\section{References}

1. Ito T, Nakano I, Koyanagi S, Miyahara T, Migita Y, Ogoshi K et al.: Autoimmune pancreatitis as a new clinical entity.

Three cases of autoimmune pancreatitis with effective steroid therapy. Dig Dis Sci 1997; 42: 1458-1468.

2. Ito T, Nishimori I, Inoue N, Kawabe K, Gibo J, Arita Y et al.: Treatment for autoimmune pancreatitis: Consensus on the treatment for patients with autoimmune pancreatitis in japan. J Gastroenterol 2007; 42 Suppl 18: 50-58.

3. Yoshida K, Toki F, Takeuchi T, Watanabe S, Shiratori K, Hayashi N: Chronic pancreatitis caused by an autoimmune abnormality. Proposal of the concept of autoimmune pancreatitis. Dig Dis Sci 1995; 40: 1561-1568.

4. Kawaguchi K, Koike M, Tsuruta K, Okamoto A, Tabata I, Fujita N: Lymphoplasmacytic sclerosing pancreatitis with cholangitis: A variant of primary sclerosing cholangitis extensively involving pancreas. Hum Pathol 1991; 22: 387-395.

5. Moon SH, Kim MH, Park DH, Hwang CY, Park SJ, Lee SS et al.: Is a 2-week steroid trial after initial negative investigation for malignancy useful in differentiating autoimmune pancreatitis from pancreatic cancer? A prospective outcome study. Gut 2008; 57: 1704-1712.

6. Steer ML, Waxman I, Freedman S: Chronic pancreatitis. N Engl J Med 1995; 332: 1482-1490.

7. Bradley EL, 3rd: Pancreatic duct pressure in chronic pancreatitis. Am J Surg 1982; 144: 313-316.

8. Kanai K, Maruyama M, Kameko F, Kawasaki K, Asano J, Oguchi T et al.: Autoimmune pancreatitis can transform into chronic features similar to advanced chronic pancreatitis with functional insufficiency following severe calcification. Pancreas 2016; 45: 1189-1195.

9. Maire F, Le Baleur Y, Rebours V, Vullierme MP, Couvelard A, Voitot H et al.: Outcome of patients with type 1 or 2 autoimmune pancreatitis. Am J Gastroenterol 2011; 106: 151-156. 
10. Maruyama M, Arakura N, Ozaki Y, Watanabe T, Ito T, Yoneda S et al.: Type 1 autoimmune pancreatitis can transform into chronic pancreatitis: A long-term follow-up study of 73 japanese patients. Int J Rheumatol 2013; 2013: 272595.

11. Maruyama M, Watanabe T, Kanai K, Oguchi T, Asano J, Ito T et al.: Autoimmune pancreatitis can develop into chronic pancreatitis. Orphanet J Rare Dis 2014; 9: 77.

12. Kawa S, Hamano H, Ozaki Y, Ito T, Kodama R, Chou Y et al.: Long-term follow-up of autoimmune pancreatitis: Characteristics of chronic disease and recurrence. Clin Gastroenterol Hepatol 2009; 7: S18-22.

13. Hart PA, Kamisawa T, Brugge WR, Chung JB, Culver EL, Czako L et al.: Long-term outcomes of autoimmune pancreatitis: A multicentre, international analysis. Gut 2013; 62: 1771-1776.

14. Ito T, Kawa S, Matsumoto A, Kubota K, Kamisawa T, Okazaki K et al.: Risk factors for pancreatic stone formation in type 1 autoimmune pancreatitis: A long-term japanese multicenter analysis of 624 patients. Pancreas 2019; 48: 49-54.

15. Maruyama M, Arakura N, Ozaki Y, Watanabe T, Ito T, Yoneda S et al.: Risk factors for pancreatic stone formation in autoimmune pancreatitis over a long-term course. J Gastroenterol 2012; 47: 553-560.

16. Uchida K, Yazumi S, Nishio A, Kusuda T, Koyabu M, Fukata M et al.: Long-term outcome of autoimmune pancreatitis. J Gastroenterol 2009; 44: 726-732.

17. Konishi K, Izumi R, Kato O, Yamaguchi A, Miyazaki I: Experimental pancreatolithiasis in the dog. Surgery 1981; 89: 687-691.

18. Sarles H, Tiscornia O, Palasciano G, Brasca A, Hage G, Devaux MA et al.: Effects of chronic intragastric ethanol administration on canine exocrine pancreatic secretion. Scand J Gastroenterol 1973; 8: 85-96.

19. Hayakawa T, Naruse S, Kitagawa M, Ishiguro H, Jin CX, Kondo T: Clinical evidence of pathogenesis in chronic 
pancreatitis. J Hepatobiliary Pancreat Surg 2002; 9: 669-674.

20. Masuda A, Shiomi H, Matsuda T, Takenaka M, Arisaka Y, Azuma T et al.: The relationship between pancreatic atrophy after steroid therapy and diabetes mellitus in patients with autoimmune pancreatitis. Pancreatology 2014; 14: 361-365.

21. Kuraishi Y, Watanabe T, Muraki T, Ashihara N, Ozawa M, Nakamura A et al.: Effectiveness of steroid therapy for pancreatic cysts complicating autoimmune pancreatitis and management strategy for cyst-related complications. Scand J Gastroenterol 2019: 1-7.

22. Asano J, Watanabe T, Oguchi T, Kanai K, Maruyama M, Ito T et al.: Association between immunoglobulin g4-related disease and malignancy within 12 years after diagnosis: An analysis after longterm followup. J Rheumatol 2015; 42: 2135-

23. Gupta R, Khosroshahi A, Shinagare S, Fernandez C, Ferrone C, Lauwers GY et al.: Does autoimmune pancreatitis increase the risk of pancreatic carcinoma?: A retrospective analysis of pancreatic resections. Pancreas 2013; 42: 506-510. 24. Miyamoto Y, Kamisawa T, Tabata T, Hara S, Kuruma S, Chiba K et al.: Short and long-term outcomes of diabetes mellitus in patients with autoimmune pancreatitis after steroid therapy. Gut Liver 2012; 6: 501-504.

25. Nishino T, Toki F, Oyama H, Shimizu K, Shiratori K: Long-term outcome of autoimmune pancreatitis after oral prednisolone therapy. Intern Med 2006; 45: 497-501.

26. Tanaka S, Kobayashi T, Nakanishi K, Okubo M, Murase T, Hashimoto M et al.: Corticosteroid-responsive diabetes mellitus associated with autoimmune pancreatitis. Lancet 2000; 356: 910-911.

27. Shimosegawa T, Chari ST, Frulloni L, Kamisawa T, Kawa S, Mino-Kenudson M et al.: International consensus diagnostic criteria for autoimmune pancreatitis: Guidelines of the international association of pancreatology. Pancreas 
2011; 40: 352-358.

28. Okazaki K, Kawa S, Kamisawa T, Ito T, Inui K, Irie H et al.: Amendment of the japanese consensus guidelines for autoimmune pancreatitis, 2013 i. Concept and diagnosis of autoimmune pancreatitis. J Gastroenterol 2014; 49: 567-588.

29. Shimosegawa T, Kataoka K, Kamisawa T, Miyakawa H, Ohara H, Ito T et al.: The revised japanese clinical diagnostic criteria for chronic pancreatitis. J Gastroenterol 2010; 45: 584-591.

30. Haaga JR, Alfidi RJ, Zelch MG, Meany TF, Boller M, Gonzalez L et al.: Computed tomography of the pancreas. Radiology 1976; 120: 589-595.

31. Naruse S, Ishiguro H, Ko SB, Yoshikawa T, Yamamoto T, Yamamoto A et al.: Fecal pancreatic elastase: A reproducible marker for severe exocrine pancreatic insufficiency. J Gastroenterol 2006; 41: 901-908.

32. Saito T, Tanaka S, Yoshida H, Imamura T, Ukegawa J, Seki T et al.: A case of autoimmune pancreatitis responding to steroid therapy. Evidence of histologic recovery. Pancreatology 2002; 2: 550-556.

33. Song MH, Kim MH, Jang SJ, Lee SK, Lee SS, Han J et al.: Comparison of histology and extracellular matrix between autoimmune and alcoholic chronic pancreatitis. Pancreas 2005; 30: 272-278.

34. Takuma K, Kamisawa T, Tabata T, Inaba Y, Egawa N, Igarashi Y: Short-term and long-term outcomes of autoimmune pancreatitis. Eur J Gastroenterol Hepatol 2011; 23: 146-152.

35. Maruyama M, Watanabe T, Kanai K, Oguchi T, Asano J, Ito T et al.: Extracorporeal shock wave lithotripsy treatment of pancreatic stones complicated with advanced stage autoimmune pancreatitis. BMC Gastroenterol 2015; $15: 28$.

36. Masamune A, Nishimori I, Kikuta K, Tsuji I, Mizuno N, Iiyama T et al.: Randomised controlled trial of long-term maintenance corticosteroid therapy in patients with autoimmune pancreatitis. Gut 2017; 66: 487-494. 
37. Nishimori I, Tamakoshi A, Kawa S, Tanaka S, Takeuchi K, Kamisawa T et al.: Influence of steroid therapy on the course of diabetes mellitus in patients with autoimmune pancreatitis: Findings from a nationwide survey in japan. Pancreas 2006; 32: 244-248. 


\section{Figure legends}

Figure 1. Prevalence of severe pancreatic calcification in autoimmune pancreatitis (AIP). (a) Flowchart of severe pancreatic calcification. Among the 145 recruited AIP patients, 19 (13\%) exhibited severe pancreatic calcification and displayed characteristic image findings fulfilling the Japanese clinical diagnostic criteria for chronic pancreatitis. Severe calcification was detected at the initial diagnosis of AIP in 2 patients and during the follow-up period after diagnosis in 17 patients. (b) CT image findings of severe pancreatic calcification. Pancreatic duct stones were seen in 12 patients. Multiple calcifications distributed throughout the entire pancreas were found in 15 patients.

Figure 2. Clinical and radiological course of a patient who received steroid therapy after detection of severe

calcification. (a) At the diagnosis of autoimmune pancreatitis, the patient exhibited diffuse pancreatic swelling, including pancreatic head swelling, following main pancreatic duct dilation. Steroid therapy was not indicated at this time. (b) Two years after diagnosis, multiple calcifications distributed throughout the pancreas were found and steroid therapy was initiated. (c) One year after starting steroid therapy, pancreatic calcifications had decreased in number. (d) Seven years after steroid therapy commencement, pancreatic calcification had remarkably improved and only a few stones remained.

Figure 3. Kaplan-Meier curves. A: The time to development of severe calcification between cases with and without steroid therapy in 143 enrolled autoimmune pancreatitis (AIP) patients. The incidence of severe calcification was significantly lower in cases with steroids than in those without (hazard ratio: $0.33,95 \%$ CI $0.12-0.94 ; \mathrm{p}=0.025, \log$-rank test). B: The time to development of severe calcification between cases with and without steroid therapy in 95 AIP patients 
with pancreatic head swelling. The incidence of severe calcification was significantly lower in cases with steroids (hazard ratio: $0.18,95 \% \mathrm{CI} 0.07-0.52 ; \mathrm{p}<0.001$, log-rank test).

Figure 4. Flowchart classification of patients based on the presence or absence of pancreatic head swelling. Two patients who already had severe calcification at the diagnosis of autoimmune pancreatitis (AIP) were excluded, and the remaining 143 AIP patients were enrolled. There were 95 patients with pancreatic head swelling and 48 patients without. Among the 95 patients with pancreatic head swelling, 77 were treated with corticosteroids and 18 were untreated, with 10 and 7 patients exhibiting severe calcification, respectively. On the other hand, no patient without pancreatic head swelling displayed severe calcification among 48 cases. 
(a)

At diagnosis

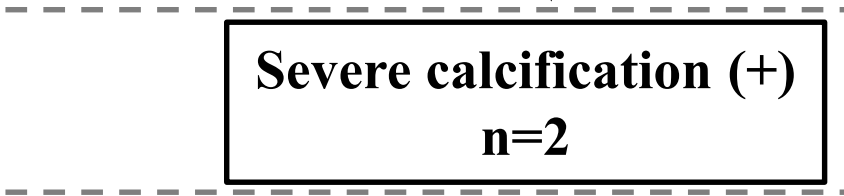

AIP patients

$$
\mathrm{n}=145
$$

Follow-up after diagnosis

Severe calcification $(+)$

$\mathrm{n}=\mathbf{1 7}$

\section{Severe calcification: $19 / 145$ cases $(13 \%)$}

(b)

Pancreatic duct stones: 12 cases
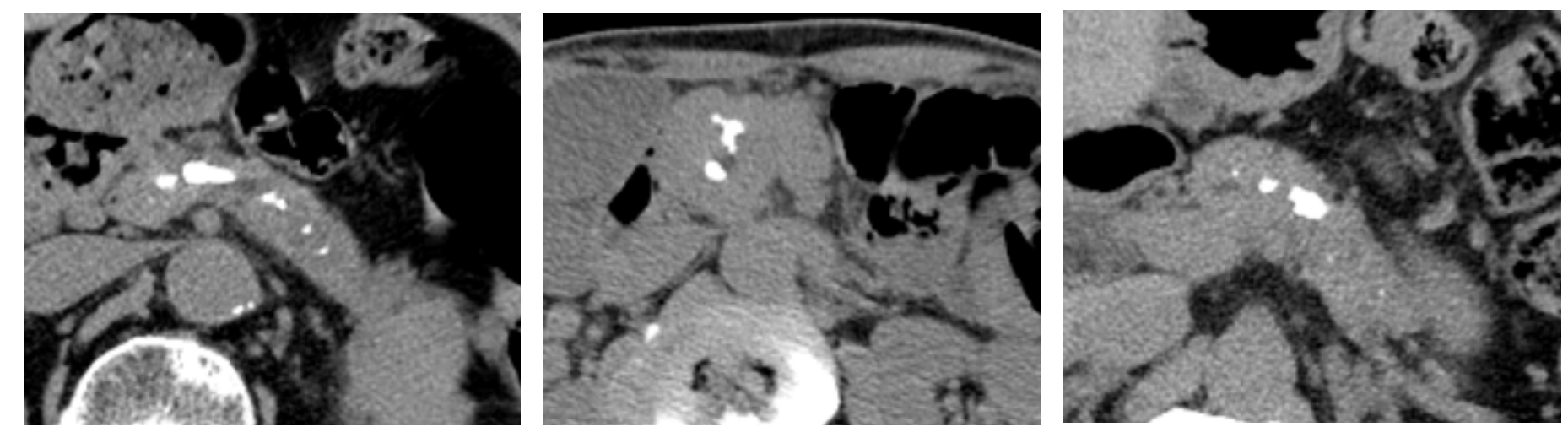

Multiple calcifications: 15 cases
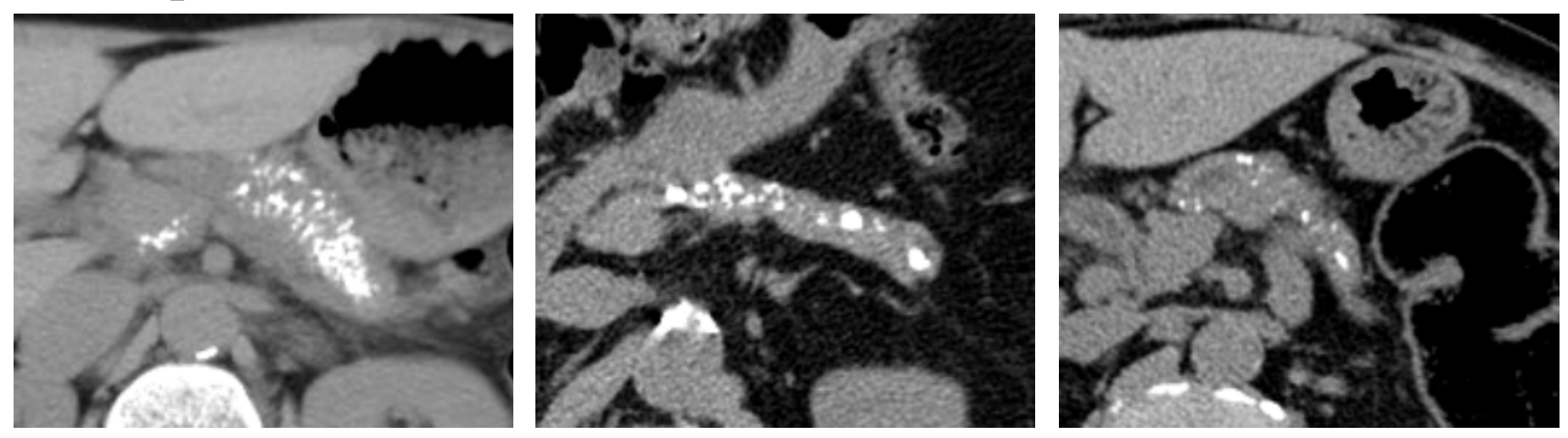
$\underline{\text { At diagnosis }}$

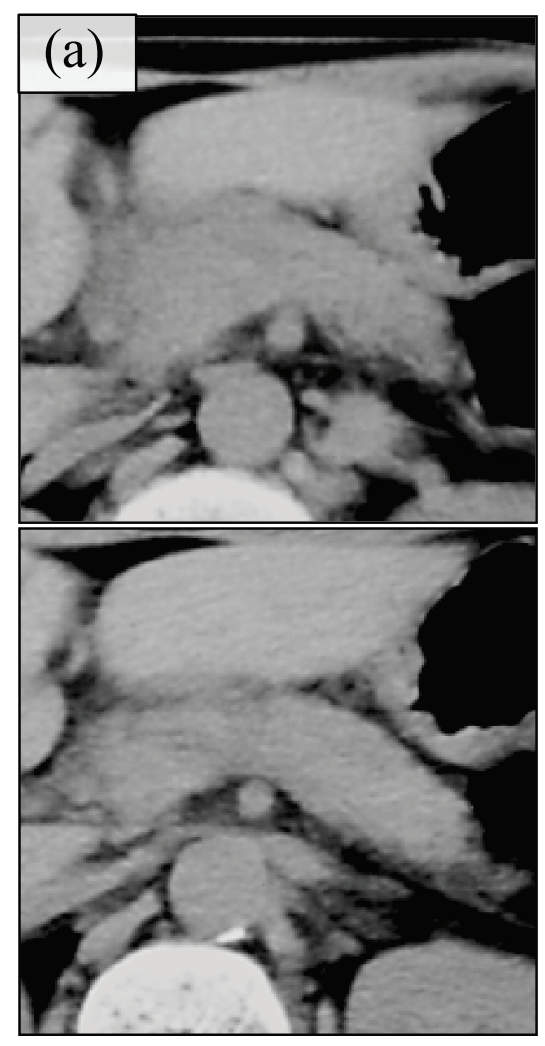

Steroid (-)
2 years

after diagnosis

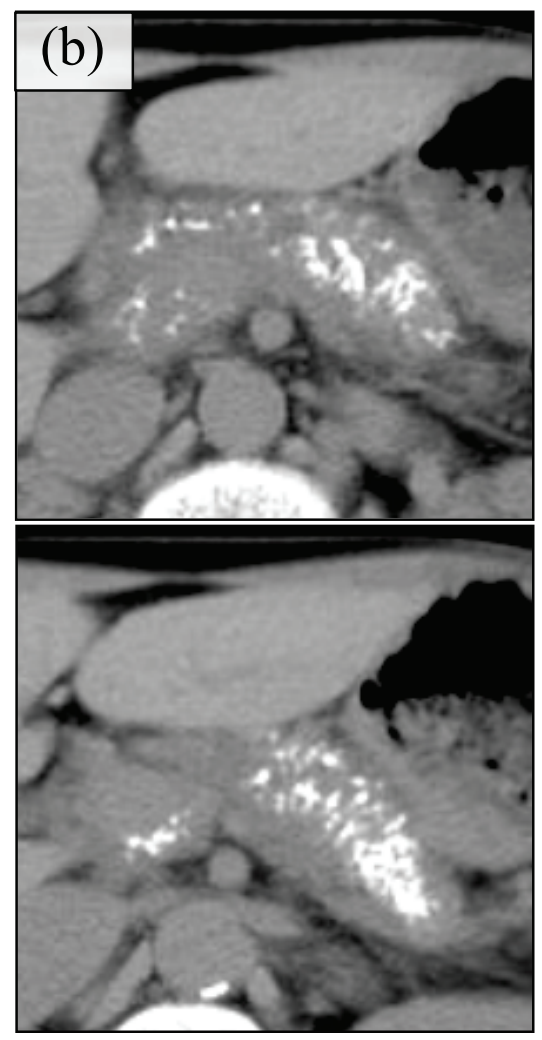

Start of steroid
1 year

after starting steroid

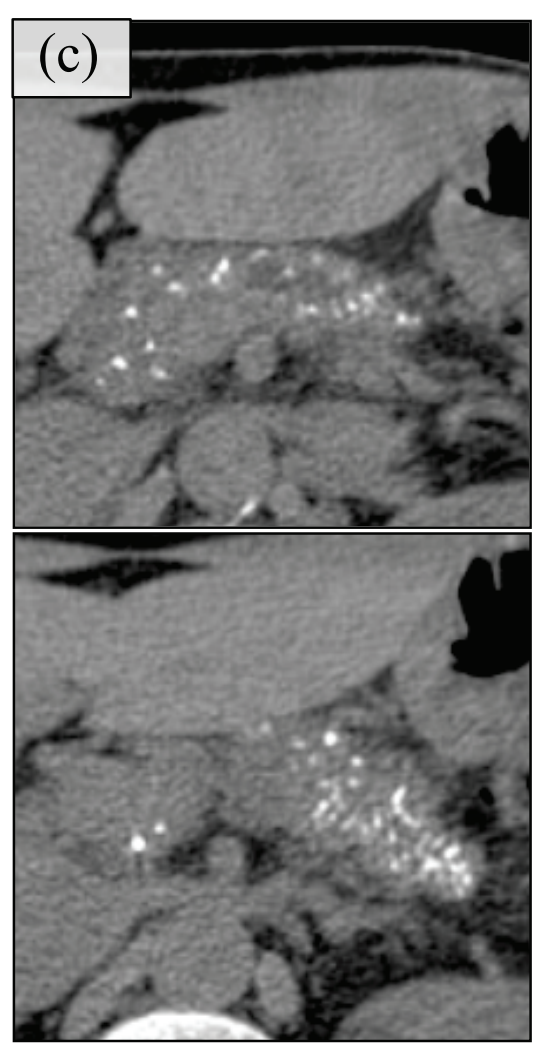

Steroid (+)
7 years

after starting steroid

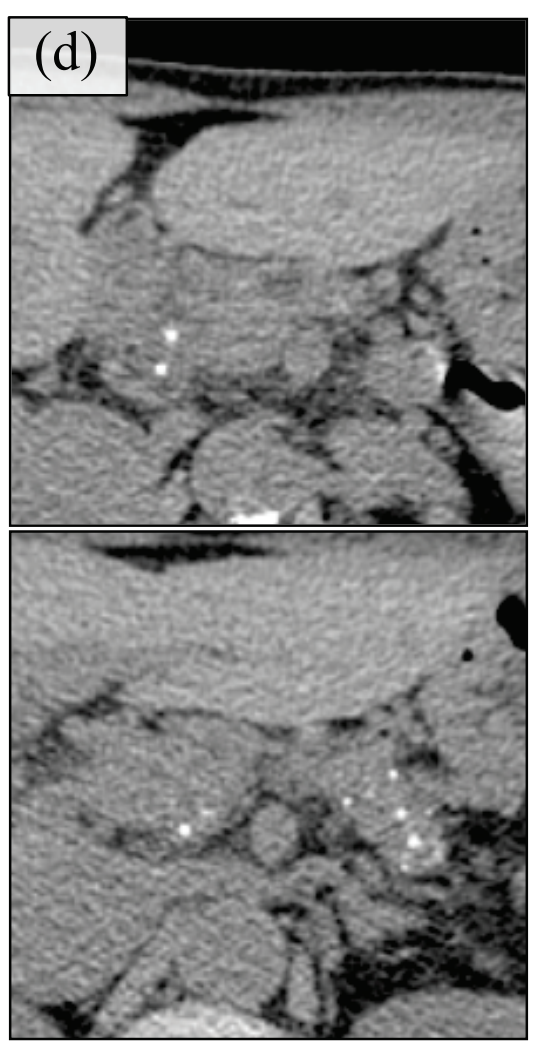

Steroid (+) 
(a)

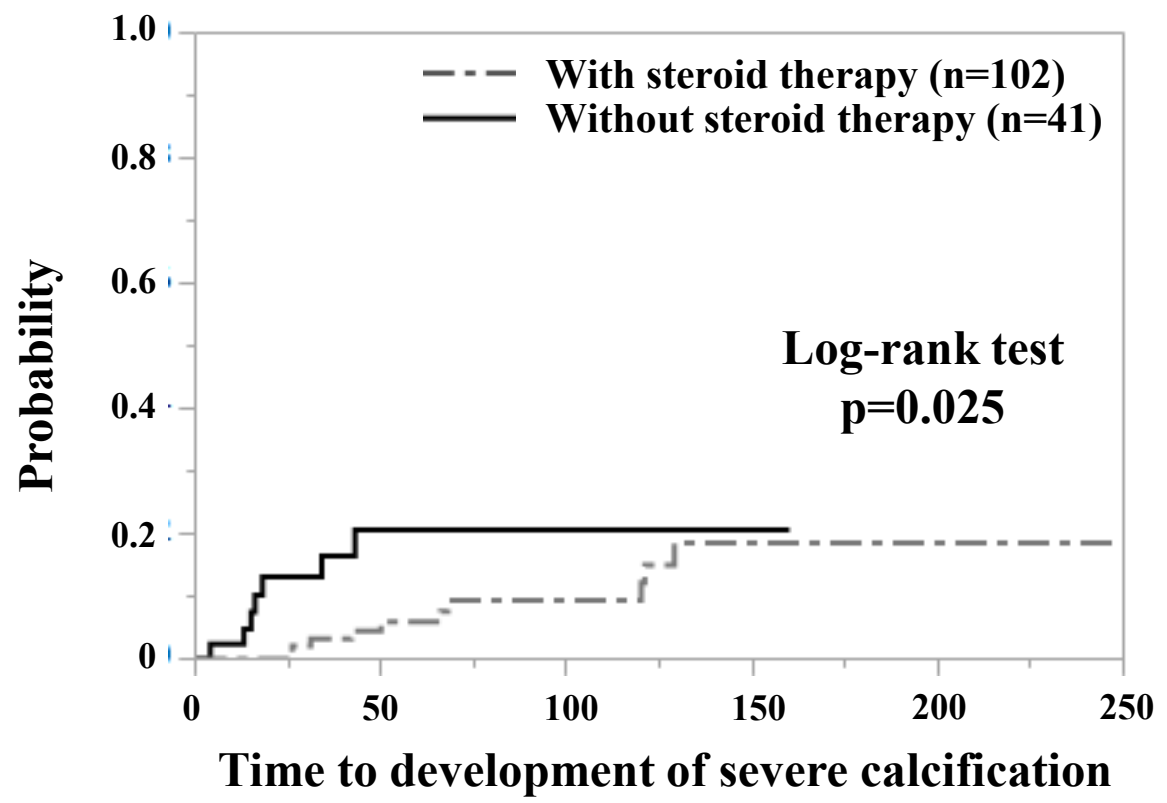

(months)

(b)

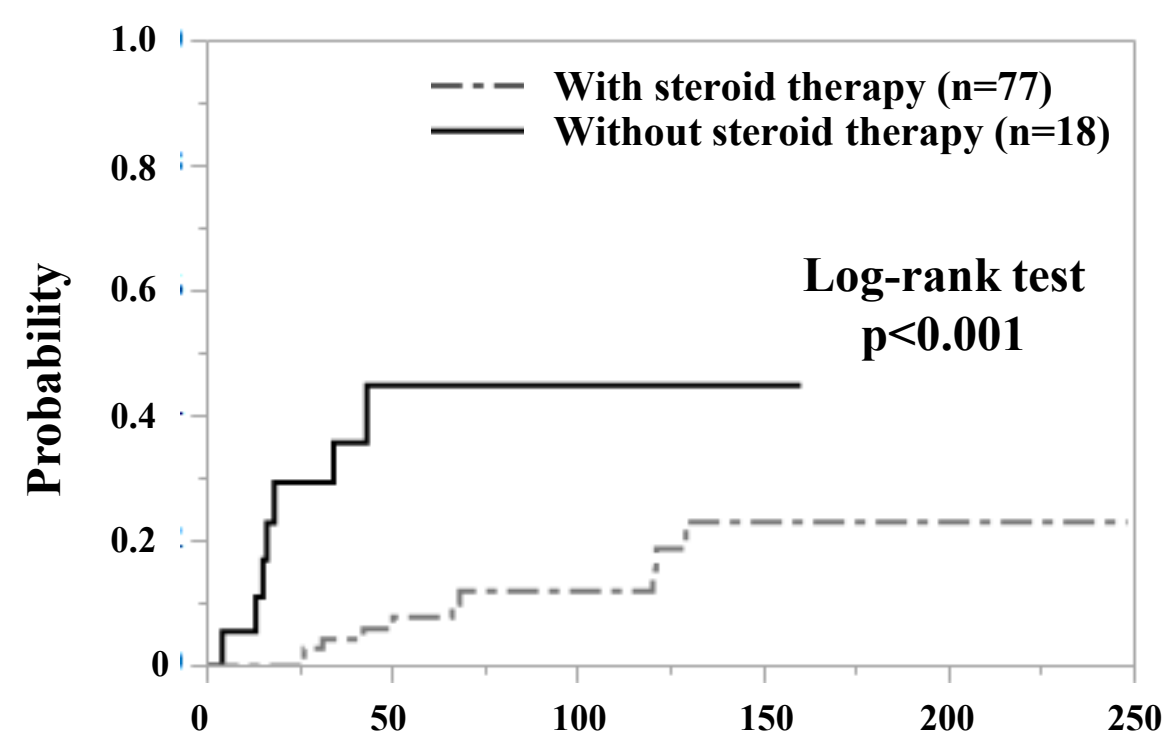

Time to development of severe calcification (months) 


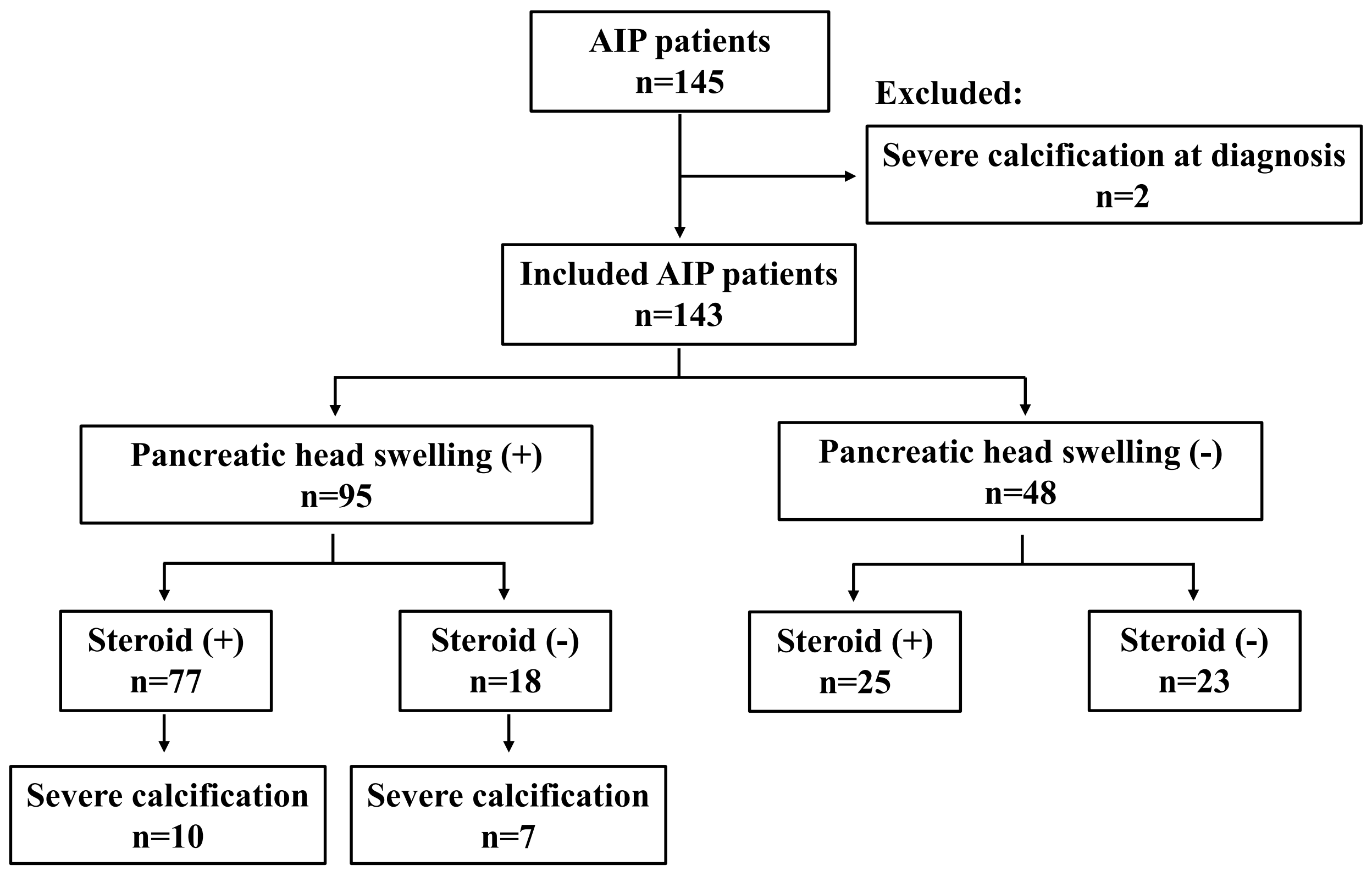




\begin{tabular}{|c|c|c|c|}
\hline \multirow[t]{2}{*}{ Factor } & \multicolumn{2}{|c|}{ Steroid therapy } & \multirow[t]{2}{*}{$\mathrm{p}$-valu } \\
\hline & $(+) \mathrm{n}=102$ & $(-) n=41$ & \\
\hline Male, n (\%) & $77(76 \%)$ & $28(68 \%)$ & 0.378 \\
\hline Age $\geq 65$ years, $n(\%)$ & $59(58 \%)$ & $24(59 \%)$ & 0.939 \\
\hline Drinking habit, n (\%) & $46(45 \%)$ & $20(49 \%)$ & 0.690 \\
\hline Smoking habit, n (\%) & $65(65 \%)$ & $20(50 \%)$ & 0.101 \\
\hline Abdominal/back pain, $\mathrm{n}(\%)$ & $39(38 \%)$ & $8(20 \%)$ & 0.031 \\
\hline Obstructive jaundice, $\mathrm{n}(\%)$ & $37(37 \%)$ & $1(3 \%)$ & $<0.001$ \\
\hline Pancreatic head swelling, n (\%) & $77(75 \%)$ & $18(44 \%)$ & $<0.001$ \\
\hline Diffuse pancreatic swelling, n (\%) & $64(63 \%)$ & $20(49 \%)$ & 0.125 \\
\hline Other organ involvement, $\mathrm{n}(\%)$ & $73(72 \%)$ & $27(66 \%)$ & 0.500 \\
\hline Serum IgG elevation, $\mathrm{n}(\%)$ & $70(69 \%)$ & $18(44 \%)$ & 0.006 \\
\hline Serum IgG4 elevation, n (\%) & $89(87 \%)$ & $40(97 \%)$ & 0.061 \\
\hline Serum amylase elevation, $\mathrm{n}(\%)$ & $28(27 \%)$ & $10(24 \%)$ & 0.708 \\
\hline MPD dilation at diagnosis, $\mathrm{n}(\%)$ & $33(32 \%)$ & $11(27 \%)$ & 0.518 \\
\hline
\end{tabular}

MPD: main pancreatic duct 
Table 2. Univariate and multivariate analysis of factors associated with severe pancreatic calcification in autoimmune pancreatitis patients with pancreatic head swelling

\begin{tabular}{|c|c|c|c|c|}
\hline \multirow[t]{2}{*}{ Factor } & \multicolumn{2}{|c|}{ Univariate analysis } & \multicolumn{2}{|c|}{ Multivariate analysis } \\
\hline & $\operatorname{HR}(95 \% \mathrm{CI})$ & $\mathrm{p}$-value & $\operatorname{HR}(95 \% \mathrm{CI})$ & p-value \\
\hline Male & $1.00(0.32-4.34)$ & 0.997 & & \\
\hline Age $\geq 65$ years & $2.04(0.76-6.08)$ & 0.160 & $2.43(0.86-7.64)$ & 0.096 \\
\hline Drinking habit & $1.13(0.43-3.04)$ & 0.790 & & \\
\hline Smoking habit & $1.16(0.36-3.33)$ & 0.787 & & \\
\hline Abdominal/back pain & $1.06(0.39-2.83)$ & 0.894 & & \\
\hline Obstructive jaundice & $0.55(0.15-1.62)$ & 0.290 & & \\
\hline Diffuse pancreatic swelling & $0.63(0.23-1.98)$ & 0.402 & & \\
\hline Steroid therapy & $0.18(0.07-0.52)$ & 0.002 & $0.11(0.03-0.34)$ & $<0.001$ \\
\hline Serum IgG elevation & $0.82(0.31-2.37)$ & 0.691 & & \\
\hline Serum IgG4 elevation & $0.71(0.04-3.57)$ & 0.734 & & \\
\hline Serum amylase elevation & $1.03(0.35-2.75)$ & 0.955 & & \\
\hline Relapse & $1.74(0.65-4.58)$ & 0.260 & & \\
\hline MPD dilation at diagnosis & $2.88(1.08-7.84)$ & 0.035 & $4.02(1.43-11.7)$ & 0.009 \\
\hline
\end{tabular}

HR: hazard ratio, 95\% CI: 95\% confidence interval, MPD: main pancreatic duct 
Table 3. Comparisons of clinical and demographic findings between autoimmune pancreatitis patients

accompanied with pancreatic head swelling and receiving steroid therapy with and without severe pancreatic calcification

\begin{tabular}{|c|c|c|c|}
\hline \multirow[t]{2}{*}{ Factor } & \multicolumn{2}{|c|}{ Severe pancreatic calcification } & \multirow[t]{2}{*}{ p-value } \\
\hline & $(+) \mathrm{n}=10$ & $(-) n=67$ & \\
\hline Male, n (\%) & $8(80 \%)$ & $52(78 \%)$ & 0.865 \\
\hline Age $\geq 65$ years, $n(\%)$ & $6(60 \%)$ & $36(53 \%)$ & 0.710 \\
\hline Drinking habit, n (\%) & $4(40 \%)$ & $31(46 \%)$ & 0.710 \\
\hline Smoking habit, $\mathrm{n}(\%)$ & $7(78 \%)$ & $44(67 \%)$ & 0.502 \\
\hline Abdominal/back pain, n (\%) & $6(60 \%)$ & $27(40 \%)$ & 0.240 \\
\hline Obstructive jaundice & $4(40 \%)$ & $30(45 \%)$ & 0.747 \\
\hline Diffuse pancreatic swelling, $\mathrm{n}(\%)$ & $7(70 \%)$ & $53(79 \%)$ & 0.517 \\
\hline Other organ involvement, $\mathrm{n}(\%)$ & $6(60 \%)$ & $45(67 \%)$ & 0.655 \\
\hline Serum IgG elevation, $\mathrm{n}(\%)$ & $7(70 \%)$ & $43(64 \%)$ & 0.719 \\
\hline Serum IgG4 elevation, $\mathrm{n}(\%)$ & $9(90 \%)$ & $57(85 \%)$ & 0.678 \\
\hline Serum amylase elevation, $\mathrm{n}(\%)$ & $4(40 \%)$ & $17(25 \%)$ & 0.333 \\
\hline MPD dilation at diagnosis, $\mathrm{n}(\%)$ & $6(60 \%)$ & $22(33 \%)$ & 0.096 \\
\hline Relapse, n (\%) & $7(70 \%)$ & $17(25 \%)$ & 0.005 \\
\hline
\end{tabular}

MPD: main pancreatic duct 